\title{
Measures of lung function and their relationship with advanced glycation end-products
}

\section{Suneela Zaigham (101, Margaretha Persson ${ }^{1}$, Amra Jujic ${ }^{1,2}$, Sophia Frantz ${ }^{3}$, Yan Borné ${ }^{1}$, Andrei Malinovschi ${ }^{4}$, Per Wollmer ${ }^{3}$ and Gunnar Engström (1) ${ }^{1}$}

Affiliations: ${ }^{1}$ Dept of Clinical Sciences Malmö, Lund University, Malmö, Sweden. ${ }^{2}$ Dept of Cardiology, Lund University, Skåne University Hospital, Malmö, Sweden. ${ }^{3}$ Dept of Translational Medicine, Lund University, Malmö, Sweden. ${ }^{4}$ Dept of Medical Sciences, Clinical Physiology, Uppsala University, Sweden.

Correspondence: Suneela Zaigham, Lund University, CRC 60:13, Jan Waldenströms gata 35, S-20502 Malmö, Sweden. E-mail: suneela.zaighamamed.lu.se

\section{ABSTRACT}

Background: Advanced glycation end-products (AGEs) have been implicated in the pathophysiology of chronic obstructive pulmonary disease (COPD). However, the association between AGE accumulation in the skin measured by skin autofluorescence (SAF) and lung function in healthy subjects has not been explored in detail. We use a population-based study of 50-64-year-olds to assess spirometry, diffusing capacity of the lung for carbon monoxide $\left(D_{\mathrm{LCO}}\right)$ and impulse oscillometry (IOS) in relation to SAF.

Methods: Participants with information on SAF, lung function and potential confounding variables were included from the Swedish Cardiopulmonary Bioimage Study (SCAPIS) cohort (spirometry, $\mathrm{n}=4111$; $D_{\text {LCO }}, \mathrm{n}=3889$; IOS, $\mathrm{n}=3970$ ). Linear regression was used to assess changes in lung function (as measured by spirometry (forced expiratory volume in $1 \mathrm{~s}\left(\mathrm{FEV}_{1}\right)$, forced vital capacity (FVC) and FEV $\left.1 / F V C\right), D_{\mathrm{LCO}}$ and IOS (resistance measured at $5\left(R_{5}\right)$ and $20 \mathrm{~Hz}\left(R_{20}\right), R_{5}-R_{20}$, area of reactance, reactance measured at $5 \mathrm{~Hz}\left(X_{5}\right)$, and resonant frequency)) by a 1-SD increase in SAF.

Results: $\mathrm{FEV}_{1}, \mathrm{FVC}$ and $D_{\mathrm{LCO}}$ were significantly and inversely associated with SAF after adjustment for potential confounding factors (per 1-SD increase in SAF: $\mathrm{FEV}_{1}-0.03 \mathrm{~L}(95 \% \mathrm{CI}-0.04--0.02 \mathrm{~L}$ ), $\mathrm{p}<0.001 ;$ FVC $-0.03 \mathrm{~L}(95 \%$ CI $-0.05--0.02 \mathrm{~L}), \mathrm{p}<0.001 ; D_{\mathrm{LCO}}-0.07 \mathrm{mmol} \cdot \mathrm{min}^{-1} \cdot \mathrm{kPa}^{-1}(95 \%$ CI $\left.\left.-0.11--0.03 \mathrm{mmol} \cdot \mathrm{min}^{-1} \cdot \mathrm{kPa}^{-1}\right), \mathrm{p}<0.001\right)$. This association was also found in nonsmokers and in non-COPD subjects. Pulmonary reactance $\left(X_{5}\right)$ but not pulmonary resistance $\left(R_{5}, R_{20}\right.$ and $\left.R_{5}-R_{20}\right)$ was significantly associated with SAF (per 1 -SD increase in SAF: $\left.X_{5}-0.001 \mathrm{kPa} \cdot \mathrm{L}^{-1} \cdot \mathrm{s}\left(95 \% \mathrm{CI}-0.003-0.00 \mathrm{kPa} \cdot \mathrm{L}^{-1} \cdot \mathrm{s}\right), \mathrm{p}=0.042\right)$, which was mirrored in non-COPD patients but not in current nonsmokers.

Conclusions: AGE accumulation, as measured by SAF, is significantly associated with lung function decrements indicative of changes in the lung parenchyma

@ERSpublications

AGEs accumulation as measured by SAF is significantly associated with lung function decrements indicative of changes in the lung parenchyma http://bit.ly/2IRgSWz

Cite this article as: Zaigham S, Persson M, Jujic A, et al. Measures of lung function and their relationship with advanced glycation end-products. ERJ Open Res 2020; 6: 00356-2019 [https:// doi.org/10.1183/23120541.00356-2019].

This article has supplementary material available from openres.ersjournals.com

Received: 18 Dec 2019 | Accepted after revision: 11 March 2020

Copyright $\odot$ ERS 2020. This article is open access and distributed under the terms of the Creative Commons Attribution Non-Commercial Licence 4.0. 


\section{Introduction}

Advanced glycation end-products (AGEs) are a group of compounds formed by a nonenzymatic reaction between reducing sugars (such as glucose) and amino acids in proteins, lipids or nucleic acids [1]. This glycation process is thought to be part of normal ageing and is known as the Maillard reaction where intermediate products, such as Schiff bases and Amadori products (e.g. glycated haemoglobin (HbA1c)), are formed, subsequently resulting in the formation of AGEs. AGEs are also formed by alternative pathways from dicarbonyl compounds that are a result of auto-oxidation and degradation products of glucose [2]. The presence and accumulation of AGEs can be measured by both circulating plasma AGEs or skin AGEs as measured by skin autofluorescence (SAF). The formation of AGEs is thought to be accelerated in chronic hyperglycaemia and oxidative stress $[2,3]$ and as such, it is well known that AGE accumulation is associated with diabetes mellitus (DM) and its complications $[1,4$, 5], including cardiovascular disease (CVD) [6-8], renal disease [4,9] and dementia [10, 11].

Plasma AGEs and SAF are thought to be increased in patients with chronic obstructive pulmonary disease (COPD) [12-15]. This relationship has been present independently of smoking, DM, CVD or renal disease [13]. SAF has also been found to be associated with a lower ratio of forced expiratory volume in $1 \mathrm{~s}$ $\left(\mathrm{FEV}_{1}\right)$ to forced vital capacity $(\mathrm{FVC})$ in patients with COPD [13]. A recent study assessed whether SAF was associated with lung function in those with normal spirometry [16]. It was found that the $\mathrm{FEV}_{1} / \mathrm{FVC}$ ratio was negatively associated with SAF only in the elderly age group with normal spirometry. Studies assessing diffusing capacity of the lung for carbon monoxide $\left(D_{\mathrm{LCO}}\right)$ and SAF have found conflicting results. A significant correlation has been found between $D_{\mathrm{LCO}}$ and SAF in a population consisting of COPD patients and healthy controls [17]. However, another study found no significant correlation between plasma AGEs or SAF and $D_{\text {LCO }}$ in patients with COPD [12].

Furthermore, the receptor for advanced glycation end-products (RAGE), which is highly expressed in the lung under normal physiological conditions [18], has been implicated in COPD development [14], COPD severity [19] and its progression [20], along with impaired spirometry and $D_{\text {LCO }}$ measurements [21]. The ligation and activation of this receptor is thought to increase an inflammatory response [22] via various pathways, including $\mathrm{NF}-\kappa \mathrm{B}$, which is responsible for chronic inflammation in many conditions. Furthermore, blockage of RAGE has also been implicated as having a protective function in COPD/ emphysema [23]. AGER is the gene that encodes RAGE, and has additionally been implicated in the susceptibility to lung function decline [24] and susceptibility to developing COPD [25]. The soluble receptor for advanced glycation end-products (sRAGE) is thought to act as a "decoy" receptor with a potential protective mechanism in which it prevents the ligation of RAGE with AGEs by clearance of circulating AGEs. The levels of sRAGE are reduced in COPD patients compared to heathy controls [26], which further reinforces a pathophysiological link between AGEs and COPD.

Although there has been much evidence to suggest a pathophysiological link between AGEs or SAF and COPD, there has been sparse literature on how changes in SAF can potentially affect lung function. To our best knowledge, there have not been any studies assessing the associations between SAF and impulse oscillometry (IOS) measurements. Measurements such as $D_{\text {LCO }}$ and IOS can give additional important information on the nature of respiratory pathology that may be present, and can potentially provide an indication of early disease that is not yet measurable by simple spirometry. The aim of the present study was to assess the association between lung function (as measured by spirometry, $D_{\text {LCO }}$ and IOS) and SAF using middle-aged subjects enrolled in a large population-based study in Sweden, the Swedish Cardiopulmonary Bioimage Study (SCAPIS).

\section{Methods}

\section{Study population}

SCAPIS is a national, multicentre, population-based study formed through collaboration between six Swedish universities (Gothenburg, Linköping, Malmö/Lund, Stockholm, Umeå and Uppsala). The project was initiated as a joint national effort to prevent CVD and COPD by improving the risk prediction of cardiopulmonary conditions using updated knowledge relevant to today's pattern of risk factors [27]. Nationwide, 30154 men and women participated. From the population of Malmö, 6251 individuals took part in the study and the participation rate in Malmö was 53\%. Study participants took part in a comprehensive questionnaire, biochemistry, extensive physical examinations, lung function tests and imaging as previously described [27]. The study was approved by the Regional Ethical Review Board in Umeå. The study participants gave their written and informed consent.

\section{Baseline examinations}

Height was measured without footwear to the nearest centimetre. Weight was measured in light indoor clothing and without footwear on a digital scale. Estimated glomerular filtration rate (eGFR) was calculated 
according to the Lund-Malmö equation [28]. Venous blood samples for immediate laboratory analysis of C-reactive protein $(\mathrm{CRP})$, plasma glucose and $\mathrm{HbA1c}$ were taken after an overnight fast $(\geqslant 8 \mathrm{~h})$. Smoking status (current, ex- or never-smoker) and pack-year history (estimated from the tobacco consumption of cigarettes, cigars and pipe tobacco) were ascertained from questionnaire responses. Prevalent DM was defined as either self-reported physician-diagnosed DM or a new diagnosis of DM at baseline. Prevalent COPD was defined as self-reported physician-diagnosed COPD, chronic bronchitis or emphysema at baseline.

\section{Lung function measurement}

Dynamic spirometry ( Jaeger MasterScreen PFT; Carefusion, Hoechberg, Germany) was performed 15 min after bronchodilation using $400 \mu \mathrm{g}$ salbutamol with subjects in the sitting position and wearing a nose clip. $\mathrm{FEV}_{1}$ and FVC were obtained according to the American Thoracic Society (ATS) and European Respiratory Society (ERS) standards [29, 30]. Reference values by Hedenström and co-workers [31, 32] were used for the calculation of $\mathrm{FEV}_{1} \%$ predicted and $\mathrm{FVC} \%$ predicted.

$D_{\text {LCO }}$ was measured using a single-breath carbon monoxide diffusion test (Jaeger MasterScreen PFT) according to ATS/ERS standards, and was performed in a sitting position and wearing a nose clip. $D_{\mathrm{LCO}}$ and alveolar volume $\left(V_{\mathrm{A}}\right)$ were calculated using the single-breath $D_{\mathrm{LCO}}$ test with helium as the tracer gas. The two measures used for further statistical analysis were $D_{\mathrm{LCO}}$ and the carbon monoxide transfer coefficient $\left(D_{\mathrm{LCO}} / V_{\mathrm{A}}\right)$. European reference values were used for the calculation of $D_{\mathrm{LCO}} \%$ predicted and $D_{\mathrm{LCO}} / V_{\mathrm{A}} \%$ predicted [33].

IOS was used for measurements for the Malmö study participants in the SCAPIS cohort (Jaeger MasterScreen PFT). Reactance and resistance were expressed in $\mathrm{kPa} \cdot \mathrm{L}^{-1} \cdot \mathrm{s}$ (area of reactance $\left(A_{X}\right)$ in $\left.\mathrm{kPa} \cdot \mathrm{L}^{-1}\right)$ and resonant frequency $\left(f_{\text {res }}\right)$ in $\mathrm{Hz}$. Examination with IOS gives a measure of respiratory impedance that is the sum of the forces that oppose pressure impulses [34] (respiratory resistance and respiratory reactance), providing information on the mechanical properties of both the central and peripheral airways [35]. It has advantages over spirometry in that no complex respiratory manoeuvre is required for measurement and it has greater sensitivity to detect peripheral airway obstruction (small airways) than spirometry [35]. Resistance at $5 \mathrm{~Hz}\left(R_{5}\right)$ is considered to be a measure of total respiratory resistance, while resistance at $20 \mathrm{~Hz}\left(R_{20}\right)$ reflects mainly the resistance of large airways. Therefore, the difference $\left(R_{5}-R_{20}\right)$ is considered to reflect more resistance in peripheral airway or ventilatory heterogeneity due to peripheral airways obstruction. Respiratory reactance is the phase shift of the input pressure signal, and consists of elastance and inertance [34]; it gives an overall measure of the respiratory system "stiffness". In COPD, there is a shift to more negative values for reactance. Reference equations by SchUlz et al. [36] were used to calculate \% predicted values for IOS measurements in this cohort.

\section{AGE measurement}

SAF (the assessment of AGE products in the skin using ultraviolet (UV) light) was performed for SCAPIS study participants in Malmö only $(n=4644)$ using an AGE reader (AGE Reader-mu Connect; DiagnOptics, Groningen, the Netherlands). A skin area of $2 \mathrm{~cm}^{2}$ on the forearm (free from scarring, tattoos and birthmarks) was illuminated with UV light $(300-420 \mathrm{~nm})$ and the light emissions from the subject's skin were registered by a spectrometer [37]. The mean of three acceptable values was taken as the final recorded result reported in arbitrary units $(\mathrm{AU})$. The procedure was noninvasive and took $\leqslant 12 \mathrm{~s}$ to complete.

\section{Analysis of data}

All analyses were carried out using SPSS version 24 (IBM Corp., Armonk, NY, USA). Subjects were included in the analysis if information on all key variables was complete: age, sex, height, weight, smoking status, pack-years, eGFR, prevalent DM, HbA1c, plasma glucose and CRP. Any potential data errors of $\mathrm{SAF}$ or lung function measures were then excluded (SAF, $\mathrm{n}=2 ; D_{\mathrm{LCO}} / V_{\mathrm{A}}, \mathrm{n}=7$; IOS measurements $\left(R_{5}\right.$, $\mathrm{n}=1$; reactance at $\left.5 \mathrm{~Hz}\left(X_{5}\right), \mathrm{n}=1 ; f_{\text {res }}, \mathrm{n}=2\right) ; A_{X}$ values $<0$ excluded, $\mathrm{n}=1 ; R_{5}-R_{20}$ values $<-0.02$ excluded, $\mathrm{n}=64$ ). Potential confounding variables with skewed distributions were log-transformed (HbA1c, plasma glucose and CRP). Three final study populations were used: subjects with information on relevant baseline examinations and either spirometry $(n=4111), D_{\text {LCO }}(n=3889)$ or IOS measures $(n=3970)$.

Linear regression was used to establish the change in the lung function measures per 1-SD increase in SAF (unstandardised $\beta$-coefficient with 95\% CI) with two models for adjustment for potential confounding variables. For the baseline table analyses, lung function variables with skewed distributions are presented as median values (\% predicted values for $R_{5}, R_{5}-R_{20}, X_{5}, A_{X}$ and $f_{\text {res }}$ ). For lung function variables with skewed distributions that could not be corrected with log-transformation (IOS measurements: $R_{5}-R_{20}, A_{X}$ and $X_{5}$ ), the bootstrapping method in linear regression was used to obtain unstandardised $\beta$-coefficients with $95 \%$ confidence intervals. The models were adjusted for confounding variables known from the literature. A p-value $<0.05$ was regarded as statistically significant. 
A sensitivity analysis was carried out in subjects who were current nonsmokers (former and never-smokers) and in subjects who had not been diagnosed with COPD.

\section{Results}

\section{Baseline characteristics}

Baseline characteristics according to tertiles of SAF are shown in table 1. Tertile (T)3 (SAF 2.2-4.6 AU) consisted of an older age group, a larger proportion of current smokers, a higher proportion of subjects with diabetes and COPD, and higher mean values of HbA1c, plasma glucose and CRP, compared to T1-T2 (SAF 1.0-2.1 AU). There was no significant association between eGFR and tertiles of SAF. Mean values of spirometry and diffusing capacity were all lower in T3 versus $\mathrm{T} 1-\mathrm{T} 2(\mathrm{p}<0.001$ for all measures). For IOS, higher \% predicted values of $X_{5}$ and $f_{\text {res }}$ were associated with higher SAF values $(\mathrm{p}=0.001$ and $\mathrm{p}<0.001$ respectively). Higher \% predicted values of IOS measures reflect an impairment in lung function.

\section{Relationship between AGEs and spirometry}

Results from linear regression models for 4111 subjects are shown in table 2. There was a significant reduction in $\mathrm{FEV}_{1}$ and $\mathrm{FVC}$ (in litres) per 1-SD increase in SAF even after full adjustments for potential confounders (per 1-SD increase in SAF: 30-mL reduction in $\mathrm{FEV}_{1}, \mathrm{p}<0.001 ; 30-\mathrm{mL}$ reduction in FVC, $\mathrm{p}<0.001$ ).

\section{TABLE 1 Baseline characteristics by tertiles (T) of skin autofluorescence ( $\mathrm{n}=4111)$}

\begin{tabular}{|c|c|c|c|c|}
\hline & $\mathrm{T} 1,1.0-1.8 \mathrm{AU}$ & $\mathrm{T} 2,1.9-2.1 \mathrm{AU}$ & $\mathrm{T} 3,2.2-4.6 \mathrm{AU}$ & p-value \\
\hline Subjects & 1480 & 1345 & 1286 & \\
\hline Age years & $56.8 \pm 4.3$ & $57.5 \pm 4.1$ & $58.4 \pm 4.3$ & $<0.001$ \\
\hline Females & $55.5 \%$ & $53.6 \%$ & $50.7 \%$ & 0.011 \\
\hline Height cm & $171.8 \pm 9.9$ & $171.7 \pm 9.6$ & $170.9 \pm 9.7$ & 0.020 \\
\hline Weight kg & $79.9 \pm 15.7$ & $80.1 \pm 15.4$ & $80.9 \pm 16.9$ & 0.087 \\
\hline \multicolumn{5}{|l|}{ Smoking } \\
\hline Current & $9.7 \%$ & $15.9 \%$ & $26.1 \%$ & $<0.001$ \\
\hline Former & $38.5 \%$ & $41.0 \%$ & $41.0 \%$ & \\
\hline Never & $51.8 \%$ & $43.0 \%$ & $32.9 \%$ & \\
\hline eGFR $\mathrm{mL} \cdot \mathrm{min}^{-1} \cdot 1.73 \mathrm{~m}^{-2}$ & $82.9 \pm 15.3$ & $82.7 \pm 14.3$ & $82.7 \pm 16.6$ & 0.643 \\
\hline Diabetes & $5.4 \%$ & $6.5 \%$ & $13.3 \%$ & $<0.001$ \\
\hline COPD & $1.5 \%$ & $1.6 \%$ & $3.3 \%$ & 0.009 \\
\hline $\mathrm{HbA} 1 \mathrm{c} \mathrm{mmol} \cdot \mathrm{mol}^{-1}$ geometric mean & 35.9 & 36.0 & 37.5 & $<0.001$ \\
\hline $\begin{array}{l}\text { Plasma glucose mmol- } \mathrm{L}^{-1} \\
\text { geometric mean }\end{array}$ & 5.38 & 5.43 & 5.58 & $<0.001$ \\
\hline CRP mmol. $\mathrm{L}^{-1}$ geometric mean & 1.3 & 1.4 & 1.6 & $<0.001$ \\
\hline $\mathrm{FEV}_{1} \mathrm{~L}$ & $3.25 \pm 0.76$ & $3.21 \pm 0.80$ & $3.06 \pm 0.76$ & $<0.001$ \\
\hline FEV $_{1} \%$ predicted & $94.9 \pm 11.6$ & $94.2 \pm 12.4$ & $92.2 \pm 12.9$ & $<0.001$ \\
\hline FVC L & $4.13 \pm 0.99$ & $4.09 \pm 1.0$ & $3.94 \pm 0.94$ & $<0.001$ \\
\hline FVC $\%$ predicted & $97.7 \pm 12.7$ & $96.6 \pm 14.1$ & $93.6 \pm 14.7$ & $<0.001$ \\
\hline $\mathrm{FEV}_{1} / \mathrm{FVC}$ & $0.790 \pm 0.06$ & $0.785 \pm 0.06$ & $0.779 \pm 0.07$ & $<0.001$ \\
\hline$D_{\text {Lco }} \%$ predicted & $90.9 \pm 12.6$ & $89.8 \pm 13.6$ & $86.9 \pm 14.6$ & $<0.001$ \\
\hline$D_{\mathrm{LCO}} / V_{\mathrm{A}} \%$ predicted & $97.0 \pm 13.5$ & $96.1 \pm 14.1$ & $94.6 \pm 15.8$ & $<0.001$ \\
\hline$R_{5} \%$ predicted & $106.9(93.3-125.6)$ & $107.7(92.1-127.8)$ & $108.6(93.3-127.3)$ & 0.312 \\
\hline$R_{20} \%$ predicted & $128.6 \pm 31.3$ & $128.5 \pm 31.1$ & $128.2 \pm 31.2$ & 0.766 \\
\hline$R_{5}-R_{20} \%$ predicted & $54.9(24.0-91.9)$ & $55.9(23.1-93.4)$ & $63.3(29.4-99.9)$ & 0.667 \\
\hline$X_{5} \%$ predicted & 87.2 (68.1-108.9) & $88.1(68.5-110.0)$ & $89.6(71.1-114.6)$ & 0.001 \\
\hline$A_{X} \%$ predicted & $60.6(38.3-97.0)$ & $61.8(39.5-101.6)$ & $65.2(39.7-112.6)$ & 0.348 \\
\hline$f_{\text {res }} \%$ predicted & $79.2(68.8-95.1)$ & $80.4(69.3-95.9)$ & $82.3(68.9-98.6)$ & $<0.001$ \\
\hline
\end{tabular}

Data are presented as mean \pm SD or median (interquartile range), unless otherwise stated. Diffusing capacity parameters: \% predicted values calculated for 3889 subjects (T1, $n=1408 ; T 2, n=1273 ; T 3, n=1208$ ). Impulse oscillometry parameters: \% predicted values presented for 3970 subjects $(T 1, n=1433 ; T 2, n=1298$; T3, $\mathrm{n}=1239$ ). $\mathrm{p}$-value: ANOVA or Chi-squared for test of linearity for continuous and categorical variables, respectively. Mean values of $X_{5}$ in T1-T3 of SAF are: T1, $-0.089 \mathrm{kPa} \cdot \mathrm{L}^{-1} \cdot \mathrm{s} ; \mathrm{T} 2,-0.092 \mathrm{kPa} \cdot \mathrm{L}^{-1} \cdot \mathrm{s} ; \mathrm{T} 3$, $-0.097 \mathrm{kPa} \cdot \mathrm{L}^{-1} \cdot \mathrm{s}$. Higher \% predicted values of impulse oscillometry measures reflect an impairment in lung function. eGFR: estimated glomerular filtration rate; HbA1c: glycated haemoglobin; CRP: C-reactive protein; $F E V_{1}$ : forced expiratory volume in $1 \mathrm{~s}$; FVC: forced vital capacity; $D_{\text {LCo: }}$ : diffusing capacity of the lung for carbon monoxide; $V_{\mathrm{A}}$ : alveolar volume; $R_{5}$ : resistance measured at $5 \mathrm{~Hz} ; R_{20}$ : resistance measured at $20 \mathrm{~Hz} ; X_{5}$ reactance measured at $5 \mathrm{~Hz} ; A_{X}$ : area of reactance; $f_{\text {res }}$ : resonant frequency. 


\begin{tabular}{|c|c|c|c|}
\hline & & Per 1-sD increase in SAF $(95 \% \mathrm{CI})$ & $\mathrm{p}$-value \\
\hline \multirow[t]{2}{*}{$\mathrm{FEV}_{1} \mathrm{~L}$} & Model 1 & $-0.06(-0.07--0.04)$ & $<0.001$ \\
\hline & Model 2 & $-0.03(-0.04--0.02)$ & $<0.001$ \\
\hline \multirow[t]{2}{*}{ FVC L } & Model 1 & $-0.05(-0.07--0.03)$ & $<0.001$ \\
\hline & Model 2 & $-0.03(-0.05--0.02)$ & $<0.001$ \\
\hline \multirow[t]{2}{*}{$\mathrm{FEV}_{1} / \mathrm{FVC}$} & Model 1 & $-0.005(-0.007--0.003)$ & $<0.001$ \\
\hline & Model 2 & $-0.002(-0.004--0.000)$ & 0.120 \\
\hline \multicolumn{4}{|c|}{$\begin{array}{l}\text { Model } 1 \text { adjusted for age, height and sex. Model } 2 \text { adjusted for age, height, sex, weight, smoking status } \\
\text { (never-, ex- and current smokers), pack-years, estimated glomerular filtration rate, diabetes, log(glycated } \\
\text { haemoglobin), log(plasma glucose) and log(C-reactive protein). FEV } 1 \text { : forced expiratory volume in } 1 \mathrm{~s} \text {; FVC: } \\
\text { forced vital capacity. }\end{array}$} \\
\hline
\end{tabular}

\section{Relationship between AGEs and diffusing capacity}

Results from linear regression models for 3889 subjects are shown in table 3. There was a significant reduction in diffusing capacity measures per 1-SD increase in SAF after full adjustments for potential confounders (per 1-SD increase in SAF: $0.07-\mathrm{mmol} \cdot \mathrm{min}^{-1} \cdot \mathrm{kPa}^{-1}$ reduction in $D_{\mathrm{LCO}}, \mathrm{p}<0.001$; $0.01-\mathrm{mmol} \cdot \mathrm{min}^{-1} \cdot \mathrm{kPa}^{-1} \cdot \mathrm{L}^{-1}$ reduction in $\left.D_{\mathrm{LCO}} / V_{\mathrm{A}}, \mathrm{p}=0.002\right)$.

\section{Relationship between AGEs and IOS measures}

Results from linear regression for 3970 subjects are shown in table 4. Per each 1-SD increase in SAF, there was a $0.001-\mathrm{kPa} \cdot \mathrm{L}^{-1} \cdot \mathrm{s}$ reduction in $X_{5}(\mathrm{p}=0.042)$ even after full adjustment for confounding variables. Per each 1-SD increase in SAF, there was a borderline significant increase in $f_{\text {res }}$ after adjusting for all potential confounding factors $\left(0.1-\mathrm{Hz}\right.$ increase in $f_{\text {res }}$ per 1 -SD increase in SAF, $\left.\mathrm{p}=0.054\right)$. The associations between other IOS measures and SAF did not remain significant after full adjustment for potential confounders.

\section{Sensitivity analyses}

A sensitivity analysis was performed in current nonsmokers (supplementary table 1). After adjustment for potential confounding factors there was a significant reduction in $\mathrm{FEV}_{1}, \mathrm{FVC}, D_{\mathrm{LCO}}$ and $D_{\mathrm{LCO}} / V_{\mathrm{A}}$ per 1 -SD increase in SAF. There was no significant association between either $\mathrm{FEV}_{1} / \mathrm{FVC}$ or IOS measures with 1 -SD increase in SAF after adjustment for potential confounding variables in nonsmokers.

Furthermore, a sensitivity analysis was performed in subjects with no known COPD diagnosis (supplementary table 2). Those with either confirmed COPD or no response/missing information for the question about prevalent COPD were excluded. A significant reduction in $\mathrm{FEV}_{1}, \mathrm{FVC}, D_{\mathrm{LCO}}, D_{\mathrm{LCO}} / V_{\mathrm{A}}$ and $X_{5}$ per each 1-SD increase in SAF remained after adjustment for confounding variables.

\section{Discussion}

In $~ 4000$ subjects aged 50-64 years old, SAF was found to have a significant inverse association with measures of spirometry, diffusing capacity and IOS measures such as $X_{5}$ even after adjustments for potential confounding variables. This inverse association between SAF and $\mathrm{FEV}_{1}$, FVC or $D_{\mathrm{LCO}}$ was consistent in separate analyses in nonsmokers or subjects without a physician-diagnosis of COPD. The ratio of $\mathrm{FEV}_{1} / \mathrm{FVC}$ was not associated with $\mathrm{SAF}$. The relationship between $X_{5}$ and SAF remained significant in subjects with no COPD diagnosis but did not remain significant in current nonsmokers.

\section{TABLE 3 Change in diffusion capacity measures per 1-SD increase in skin autofluorescence (SAF) (n=3889)}

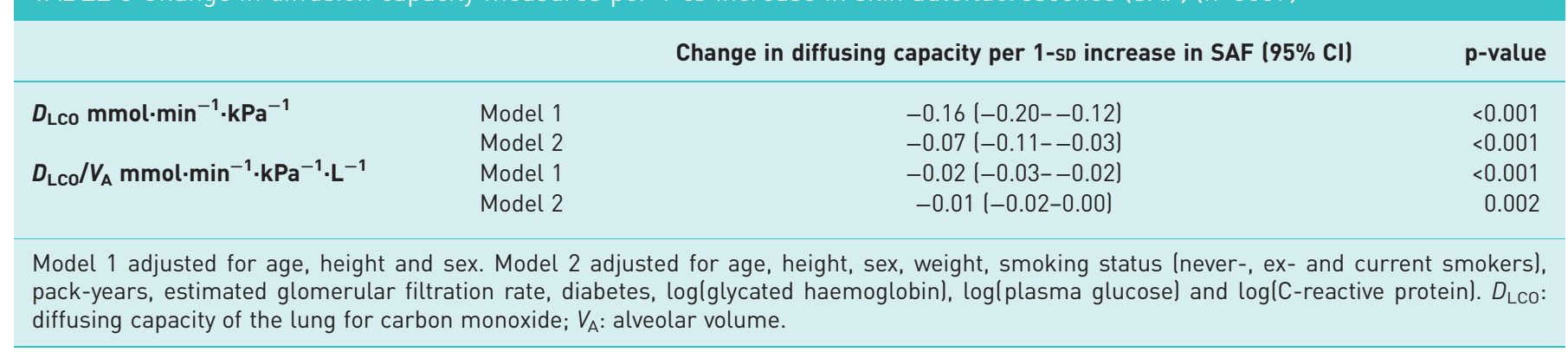




\begin{tabular}{|c|c|c|c|}
\hline & & Change in IOS measure per $1-\mathrm{SD}$ increase in SAF $(95 \% \mathrm{CI})$ & p-value \\
\hline \multirow[t]{2}{*}{$R_{5} \mathrm{kPa} \cdot \mathrm{L}^{-1} \cdot \mathrm{s}$} & Model 1 & $0.003(0.00-0.006)$ & 0.044 \\
\hline & Model 2 & $-0.001(-0.003-0.002)$ & 0.628 \\
\hline \multirow{2}{*}{$R_{20} \mathrm{kPa} \cdot \mathrm{L}^{-1} \cdot \mathrm{s}$} & Model 1 & $0.00(-0.002-0.003)$ & 0.732 \\
\hline & Model 2 & $-0.001(-0.003-0.001)$ & 0.337 \\
\hline \multirow{2}{*}{$R_{5}-R_{20} \mathrm{kPa} \cdot \mathrm{L}^{-1} \cdot \mathrm{s}$} & Model 1 & $0.002(0.001-0.004)$ & 0.002 \\
\hline & Model 2 & $0.00(-0.001-0.002)$ & 0.509 \\
\hline \multirow[t]{2}{*}{$A_{X} \mathrm{kPa} \cdot \mathrm{L}^{-1}$} & Model 1 & $0.020(0.009-0.031)$ & 0.001 \\
\hline & Model 2 & $0.006(-0.003-0.015)$ & 0.229 \\
\hline \multirow[t]{2}{*}{$f_{\text {res }} \mathrm{Hz}$} & Model 1 & $0.253(0.150-0.357)$ & $<0.001$ \\
\hline & Model 2 & $0.100(-0.002-0.199)$ & 0.054 \\
\hline \multirow[t]{2}{*}{$X_{5} \mathrm{kPa} \cdot \mathrm{L}^{-1} \cdot \mathrm{s}$} & Model 1 & $-0.003(-0.004--0.002)$ & 0.001 \\
\hline & Model 2 & $-0.001(-0.003--0.00006)$ & 0.042 \\
\hline
\end{tabular}

There is a known decline in $\mathrm{FEV}_{1}$ and FVC with normal ageing, and AGEs accumulate with normal ageing. Similarly, smoking is associated with both a decline in $\mathrm{FEV}_{1}$ and accumulation of AGEs. However, a significant inverse association remained even after taking into account these two major factors common to the worsening of both lung function and increase in SAF. $D_{\mathrm{LCO}}$ assesses the gas exchanging characteristics of the lung parenchyma, and is used mainly in the diagnosis and management of diseases of the lung parenchyma such as emphysema [33]. The association between $D_{\text {LCO }}$ and SAF was significant in our study, even after adjustments, and could be found for both $D_{\mathrm{LCO}}$ and $D_{\mathrm{LCO}} / V_{\mathrm{A}}$, suggesting that there is a relationship between SAF and impaired alveolocapillary membranes. Previous studies assessing AGEs and $D_{\text {LCO }}$ have been carried out with conflicting results. One study found negative correlation between $D_{\mathrm{LCO}} \%$ predicted and SAF $\left(\mathrm{r}^{2}=0.276, \mathrm{p}<0.0001\right)$ [17] in a cohort of COPD patients and healthy controls $(\mathrm{n}=288)$, whereas another found no correlation between $D_{\mathrm{LCO}}$ and SAF $(\mathrm{r}=0.113, \mathrm{p}=0.33)$ in 88 COPD patients [12]. We found a significant inverse association between $D_{\mathrm{LCO}}$ and SAF in our overall cohort, and in the sensitivity analysis where known COPD subjects were excluded. With regards to IOS, respiratory reactance, but not resistance, was associated with SAF as indicated by a significant inverse association between SAF and $X_{5}$, and a lack of association between $R_{5}, R_{20}$ or $R_{5}-R_{20}$ and SAF. In COPD patients, measures of reactance such as $X_{5}$ have been found to be indicative of changes in the elastance of the respiratory system that can be caused by increased heterogeneity, as occurs in airways obstruction [38]. Moreover, bronchodilators improve $X_{5}$ in COPD, likely reflecting the improvement of airways obstruction and, subsequently, ventilation heterogeneity [39]. Although the ratio of $\mathrm{FEV}_{1} / \mathrm{FVC}$ was not associated with SAF, we found that $X_{5}$ was associated with SAF even after adjustment for confounders (and even in non-COPD subjects). This finding can also, to some extent, indicate an association between SAF and airways obstruction.

Both circulating plasma AGEs and SAF accumulation have been found to be associated with conditions related to chronic hyperglycaemia or oxidative stress, including DM, CVD, renal failure and dementia. However, the association with SAF and lung function has not previously been assessed in detail, and many of the existing studies compare AGEs in subjects with COPD versus subjects without COPD. A recent study assessed the association between SAF and spirometry [16], and found a correlation between $\mathrm{FEV}_{1} / \mathrm{FVC}$ and SAF in elderly subjects (age 69 \pm 7 years; $\mathrm{r}=-0.446, \mathrm{p}=0.002$ ) but not in younger subjects (aged $51 \pm 8$ years). The study cohort consisted of those with normal spirometry at baseline. Therefore, these findings can be compared to our results from a subcohort of subjects not known to be diagnosed with COPD. The age range in the SCAPIS cohort was comparable to this younger cohort; as such, we mirror the findings that at this "younger" cohort age range, SAF was not associated with $\mathrm{FEV}_{1} / \mathrm{FVC}$. Future research should examine whether raised SAF levels in apparently healthy subjects are associated with increased risk of developing COPD.

Our results indicate an association between SAF and changes in the lung parenchyma, as indicated by the significant inverse associations between $D_{\text {LCO }}$ and SAF, even in sensitivity analyses. It remains unclear whether these changes would be emphysematous or fibrotic in nature. However, the lack of association between the $\mathrm{FEV}_{1} / \mathrm{FVC}$ ratio and $\mathrm{SAF}$, and the significant relationships between lung function measures and SAF in a subcohort of non-COPD subjects would indicate that in this cohort, it is unlikely that 
emphysematous changes in the parenchyma would fully explain the associations between SAF and $D_{\text {LCO. }}$ Our results potentially indicate an association between a restrictive lung defect and SAF; however, we had no information on total lung capacity (TLC) to confirm this.

The association between restrictive lung diseases such as idiopathic pulmonary fibrosis (IPF) and serum AGEs has also been explored, where it has been found that those with IPF have increased levels of serum AGEs [40, 41]. It has been found that in IPF patients, a change in serum AGEs over time corresponds to a decline in lung function over time; for example, a reduction in $D_{\mathrm{LCO}} \%$ predicted, FVC and TLC was associated with an increase in serum AGEs [41]. Although it remains unclear if SAF is only a result of locally formed skin AGEs or whether increased SAF can also occur from accumulation from AGEs in the circulation, there seems to be some emerging evidence supporting the latter. A study in 2014 assessed the role of both plasma AGEs and SAF in COPD patients, and found that in addition to both plasma AGE levels and SAF being associated with COPD, certain plasma protein-bound AGEs were also associated with SAF [12]. As the evidence is still very limited, the precise mechanisms by which skin AGE accumulation is associated with lung function can only be speculated. It has been hypothesised that continuous exposure to oxidative stress, such as in conditions like COPD, may lead to the accumulation of AGEs both inside and outside the lung [17]. The level of the protective decoy receptor sRAGE has been found to be reduced in both COPD patients [42] and patients with IPF [41]. Furthermore, it has been thought that an increase in the AGE/sRAGE ratio could also indicate the degree of remodelling due to pulmonary fibrosis [41]. Interestingly, it has also been found that lower levels of sRAGE are associated with higher SAF in COPD subjects, the lack of decoy receptor potentially then resulting in accumulation of AGEs in the skin. This hypothesis could also be extended to the association between sRAGE and SAF in IPF patients as a potential mechanism for the findings linking SAF to parenchymal lung diseases.

\section{Study limitations}

Although a significant association was found between certain lung measures and SAF, we are aware that the effect sizes per 1-SD increase in SAF were not large. We appreciate that as such, the clinical relevance may be uncertain. Nevertheless, the highly significant p-values for associations (especially $\mathrm{FEV}_{1}$, FVC, $D_{\mathrm{LCO}}$ and $D_{\mathrm{LCO}} / V_{\mathrm{A}}$ with $\mathrm{SAF}$ ) indicate that although small, a negative association between lung function and SAF was found in this cohort. The presence of an association in a "healthy" cohort such as SCAPIS can potentially provide some insight into how this association may potentially be more pronounced in a more "unhealthy" cohort and highlight the need for longitudinal studies to assess the direction of this small but significant association. The study population consisted of 50-64-year-old subjects; therefore, the findings may not be readily generalisable to younger age groups. However, as the accumulation of AGEs and decline in lung function are generally processes that occur at an older age, these findings may be of greater significance in the 50-64-year-old age group. Study participants were mainly of European origin living in Malmö, Sweden, which again, would limit the generalisability of the results to other populations. In observational studies of this nature, there is also a degree of selection bias to be considered. Those who took part in the study may differ in lifestyle habits from those that declined the invitation to be part of the study. There may therefore be an element of a healthier population agreeing to be part of such a study. However, $\sim 17 \%$ of the participants were current smokers, which is comparable to the prevalence of smoking in Sweden (14.7\% of men and women aged 55-64 years in 2018) [43]. Due to the nature of this observational cross-sectional study, there may also be the potential for residual confounding in the relationship between SAF and lung function. To take into account the role of inflammation in the association between SAF and low levels of lung function, we adjusted for baseline levels of CRP in our analysis, and found an association to exist between SAF and spirometry, $D_{\mathrm{LCO}}$ and $X_{5}$ despite this adjustment. However, the role of inflammation could not be fully excluded, as RAGE activation has been implicated with various inflammatory mediators (e.g. NF- $\mathrm{KB})$; therefore, inflammation could still, in part, explain the associations observed. Chronic oxidative stress is an important accelerator of AGE formation and is associated with lung diseases such as COPD [17]. Even though many of the adjustment variables in this study are related to oxidative stress to some extent, we had no possibility to specifically adjust the results for oxidative stress. Hence, the role of oxidative stress cannot be excluded. Future studies should directly consider the role of parameters of oxidative stress on the association between SAF and lung function. Although the results indicate a potential relationship between SAF and restrictive lung defect, we did not have information on TLC; hence, we cannot conclusively state an association does indeed exist. Future studies are needed in which total lung volume on computerised tomography (as a proxy for TLC) or from body plethysmography can be used to confirm these findings.

We adjusted for plasma glucose, HbAlc and a diagnosis of DM. Glucose is part of the causal chain leading to the formation of AGEs and, as such, this could be considered as over-adjustment of the relationship between SAF and lung function. However, it is noteworthy that the relationships between SAF and lung function, in most cases, persisted after extensive adjustment for glucose. 


\section{Study strengths}

This study does, however, have many strengths. Post-bronchodilator measurements were used,which are advocated for the diagnosis of COPD [44], to ensure an element of reversibility (e.g. due to asthma) can be ruled out. The large sample size of the SCAPIS cohort is also an additional strength, adding power to our significant findings. To our knowledge, this is the first population-based study to assess the relationship between AGE accumulation in the skin with extensive lung function assessed according to standardised protocols including spirometry, $D_{\mathrm{LCO}}$ and IOS measures in a healthy population of 50-64-year-olds.

\section{Conclusions}

An increase in AGE accumulation, as measured by SAF, is significantly associated with lung function decrements indicative of changes in the lung parenchyma.

Conflict of interest: S. Zaigham has nothing to disclose. M. Persson has nothing to disclose. A. Jujic has nothing to disclose. S. Frantz has nothing to disclose. Y. Borné has nothing to disclose. A. Malinovschi has nothing to disclose. P. Wollmer reports grants from the Swedish Heart and Lung Foundation, Skåne Region and Lund University, during the conduct of the study; and personal fees from AstraZeneca and Chiesi Pharmaceuticals, outside the submitted work. G. Engström has nothing to disclose.

Support statement: The main funding body of The Swedish Cardiopulmonary Bioimage Study (SCAPIS) is the Swedish Heart and Lung Foundation (grant 2016-0315). The study is also funded by the Knut and Alice Wallenberg Foundation (2014-0047), the Swedish Research Council (822-2013-2000), VINNOVA (Sweden's innovation agency) (2012-04476), the University of Gothenburg and Sahlgrenska University Hospital, Karolinska Institutet and Stockholm county council, Linköping University and University Hospital, Lund University and Skåne University Hospital, Umeå University and University Hospital, Uppsala University and University Hospital. Funding information for this article has been deposited with the Crossref Funder Registry.

\section{References}

$1 \quad$ Singh R, Barden A, Mori T, et al. Advanced glycation end-products: a review. Diabetologia 2001; 44: 129-146.

2 Rhee SY, Kim YS. The role of advanced glycation end products in diabetic vascular complications. Diabetes Metab J 2018; 42: 188-195.

3 Vlassara H, Uribarri J. Advanced glycation end products (AGE) and diabetes: cause, effect, or both? Curr Diab Rep 2014; 14: 453.

4 Brownlee M. Lilly Lecture 1993. Glycation and diabetic complications. Diabetes 1994; 43: 836-841.

5 Osawa S, Katakami N, Sato I, et al. Skin autofluorescence is associated with vascular complications in patients with type 2 diabetes. J Diabetes Complicat 2018; 32: 839-844.

6 Kume S, Takeya M, Mori T, et al. Immunohistochemical and ultrastructural detection of advanced glycation end products in atherosclerotic lesions of human aorta with a novel specific monoclonal antibody. Am J Pathol 1995; 147: 654-667.

7 de Vos LC, Noordzij MJ, Mulder DJ, et al. Skin autofluorescence as a measure of advanced glycation end products deposition is elevated in peripheral artery disease. Arterioscler Thromb Vasc Biol 2013; 33: 131-138.

8 Baynes JW, Thorpe SR. Glycoxidation and lipoxidation in atherogenesis. Free Radic Biol Med 2000; 28: $1708-1716$.

9 Noordzij MJ, Lefrandt JD, Smit AJ. Advanced glycation end products in renal failure: an overview. J Ren Care 2008; 34: 207-212.

10 Sasaki N, Fukatsu R, Tsuzuki K, et al. Advanced glycation end products in Alzheimer's disease and other neurodegenerative diseases. Am J Pathol 1998; 153: 1149-1155.

11 Ko SY, Ko HA, Chu KH, et al. The possible mechanism of advanced glycation end products (AGEs) for Alzheimer's disease. PLoS One 2015; 10: e0143345.

12 Gopal P, Reynaert NL, Scheijen JL, et al. Plasma advanced glycation end-products and skin autofluorescence are increased in COPD. Eur Respir J 2014; 43: 430-438.

13 Hoonhorst SJM, Lo Tam Loi AT, Hartman JE, et al. Advanced glycation end products in the skin are enhanced in COPD. Metab Clin Exp 2014; 63: 1149-1156.

14 Wu L, Ma L, Nicholson LF, et al. Advanced glycation end products and its receptor (RAGE) are increased in patients with COPD. Respir Med 2011; 105: 329-336.

15 John M, McKeever TM, Haddad MA, et al. Traditional and emerging indicators of cardiovascular risk in chronic obstructive pulmonary disease. Chron Respir Dis 2016; 13: 247-255.

16 Kubo A, Kato M, Sugioka Y, et al. Relationship between advanced glycation end-product accumulation in the skin and pulmonary function. J Phys Ther Sci 2018; 30: 413-418.

17 Hoonhorst SJ, Lo Tam Loi AT, Pouwels SD, et al. Advanced glycation endproducts and their receptor in different body compartments in COPD. Respir Res 2016; 17: 46.

18 Buckley ST, Ehrhardt C. The receptor for advanced glycation end products (RAGE) and the lung. J Biomed Biotechnol 2010; 2010: 917108.

19 Chen L, Liu L, Wang T, et al. Receptor for advanced glycation end products: a new theraputic target for chronic obstructive pulmonary disease? Arch Med Res 2013; 44: 75-76.

20 Coxson HO, Dirksen A, Edwards LD, et al. The presence and progression of emphysema in COPD as determined by CT scanning and biomarker expression: a prospective analysis from the ECLIPSE study. Lancet Respir Med 2013; 1: 129-136.

21 Cockayne DA, Cheng DT, Waschki B, et al. Systemic biomarkers of neutrophilic inflammation, tissue injury and repair in COPD patients with differing levels of disease severity. PLoS One 2012; 7: e38629. 

40-49.

23 Lee H, Park JR, Kim WJ, et al. Blockade of RAGE ameliorates elastase-induced emphysema development and progression via RAGE-DAMP signaling. FASEB J 2017; 31: 2076-2089.

24 Repapi E, Sayers I, Wain LV, et al. Genome-wide association study identifies five loci associated with lung function. Nat Genet 2010; 42: 36-44.

25 Castaldi PJ, Cho MH, Litonjua AA, et al. The association of genome-wide significant spirometric loci with chronic obstructive pulmonary disease susceptibility. Am J Respir Cell Mol Biol 2011; 45: 1147-1153.

26 Smith DJ, Yerkovich ST, Towers MA, et al. Reduced soluble receptor for advanced glycation end-products in COPD. Eur Respir J 2011; 37: 516-522.

27 Bergström G, Berglund G, Blomberg A, et al. The Swedish CArdioPulmonary BioImage Study: objectives and design. J Intern Med 2015; 278: 645-659.

28 Nyman U, Grubb A, Larsson A, et al. The revised Lund-Malmo GFR estimating equation outperforms MDRD and CKD-EPI across GFR, age and BMI intervals in a large Swedish population. Clin Chem Lab Med 2014; 52: $815-824$.

29 Miller MR, Hankinson J, Brusasco V, et al. Standardisation of spirometry. Eur Respir J 2005; 26: 319-338.

30 Miller MR, Crapo R, Hankinson J, et al. General considerations for lung function testing. Eur Respir J 2005; 26: $153-161$.

31 Hedenstrom H, Malmberg P, Fridriksson HV. Reference values for lung function tests in men: regression equations with smoking variables. Ups J Med Sci 1986; 91: 299-310.

32 Hedenstrom H, Malmberg P, Agarwal K. Reference values for lung function tests in females. Regression equations with smoking variables. Bull Eur Physiopathol Respir 1985; 21: 551-557.

33 Cotes JE, Chinn DJ, Quanjer PH, et al. Standardization of the measurement of transfer factor (diffusing capacity). Eur Respir J 1993; 6: Suppl. 16, 41-52.

34 Bickel S, Popler J, Lesnick B, et al. Impulse oscillometry: interpretation and practical applications. Chest 2014; 146: $841-847$.

35 Brashier B, Salvi S. Measuring lung function using sound waves: role of the forced oscillation technique and impulse oscillometry system. Breathe 2015; 11: 57-65.

36 Schulz H, Flexeder C, Behr J, et al. Reference values of impulse oscillometric lung function indices in adults of advanced age. PLoS One 2013; 8: e63366-e63366.

37 Meerwaldt R, Graaff R, Oomen PHN, et al. Simple non-invasive assessment of advanced glycation endproduct accumulation. Diabetologia 2004; 47: 1324-1330.

38 Kolsum U, Borrill Z, Roy K, et al. Impulse oscillometry in COPD: identification of measurements related to airway obstruction, airway conductance and lung volumes. Respir Med 2009; 103: 136-143.

39 Borrill ZL, Houghton CM, Woodcock AA, et al. Measuring bronchodilation in COPD clinical trials. Br J Clin Pharmacol 2005; 59: 379-384.

40 Machahua C, Montes-Worboys A, Llatjos R, et al. Increased AGE-RAGE ratio in idiopathic pulmonary fibrosis Respir Res 2016; 17: 144-144.

41 Machahua C, Montes-Worboys A, Planas-Cerezales L, et al. Serum AGE/RAGEs as potential biomarker in idiopathic pulmonary fibrosis. Respir Res 2018; 19: 215.

42 Gopal P, Reynaert NL, Scheijen JL, et al. Association of plasma sRAGE, but not esRAGE with lung function impairment in COPD. Respir Res 2014; 15: 24.

43 SCB: Statistics Sweden. Tobacco habits by indicator, age and sex. 2008-2018. www.statistikdatabasen.scb.se/pxweb/ sv/ssd/START_LE_LE0101__LE0101H/LE0101H25/?rxid=a47ef711-0c9f-4281-9ea3-7bd54ec8e9a2

44 Global Initiative for Chronic Obstructive Lung Disease. Global strategy for the diagnosis, management and prevention of chronic obstructive pulmonary disease, 2018 report. http://goldcopd.org/wp-content/uploads/2017/ 11/GOLD-2018-v6.0-FINAL-revised-20-Nov_WMS.pdf 